\title{
Distribution of basement membrane antigens in clinical gastric adenocarcinomas: an immunohistochemical study
}

\author{
K NAKAMURA, M MORI, M ENJOJI \\ From the Second Department of Pathology, Faculty of Medicine, Kyushu University, Fukuoka, Japan
}

SUMMARY The distribution of laminin and collagen type IV in the basement membranes of 85 gastric adenocarcinomas was studied using immunoperoxidase techniques to check for invasive carcinoma. Lymph nodes with metastases were also studied in 23 cases. Thick and discontinuous staining of the basement membranes was observed in 12 cases of well differentiated adenocarcinoma; thin and discontinuous staining in 26 (12 well and 14 moderately differentiated adenocarcinomas); fragmentary staining in 36 (15 moderately and 21 poorly differentiated adenocarcinomas); and unrecognisable basement membrane staining in the remaining 11 cases of poorly differentiated adenocarcinoma. These patterns were largely related to the histological grade, the nuclear atypism and loss of polarity of tumour cells, and the degree of inflammatory infiltration.

Basement membranes are complex structures lining epithelia on connective tissue stroma. ${ }^{12}$ Distribution of these tissues differs in various states, particularly in neoplastic lesions. ${ }^{2-5}$ Laminin and collagen type IV are natural basement membrane components, ${ }^{6}$ and their distributions have been well studied in carcinomas of various organs. ${ }^{7-9}$ Recent reviews have shown that the changes in basement membrane distribution are related to the histological grade and metastatic property of tumours; this has prognostic implications. ${ }^{10-13}$ We analysed immunohistochemically the distributions of basement membranes in serial tissue sections, expressed by the reactivity to laminin and collagen type IV in human gastric adenocarcinomas.

\section{Material and methods}

Stomachs were resected from 85 patients $(61$ men, 24 women, age range 23-81 years) with a carcinoma, including 23 with spread to the regional lymph nodes. All but two patients were available for follow up data. Each specimen was fixed in buffered formalin, cut into slices, embedded in paraffin, and cut into three serial $5 \mu \mathrm{m}$ thick sections. One section was stained with haematoxylin and eosin to confirm the histopathological diagnosis, and the other two were treated with $0.4 \%$ pepsin (Sigma P-7012) in 0.09 $\mathrm{N}$ hydrochloric acid for two hours at $37^{\circ} \mathrm{C}$, washeds and reacted with rabbit antibodies against mouse laminin (Bethesda Research Laboratories, Gaithersburg, Maryland, USA) and with mouse antibodies against human collagen type IV (Australian Monoclonal Development, Artarmon, Australia), respectively. Both antisera were used after diluting $1 / 50$ in phosphate buffered saline at a $\mathrm{pH}$ of 7.3.

Bound antibody was detected by the avidin-biotinperoxidase complex (ABC) method of Hsu et al ${ }^{14}$ after reaction with $0.2 \%$ hydrogen peroxide to remove endogenous peroxidase. Sections were treated with biotinylated antirabbit (for laminin staining) or antimouse (for collagen type IV staining), immunoglobulin antiserum $(1 / 500)$, avidin $(1 / 1000)$, and biotinylated horseradish peroxidase complex (Vector Laboratories, Burlingame, California, USA). The peroxidase was developed with $0.01 \% \quad \mathrm{H}_{2} \mathrm{O}_{2}$ and $0.05 \%$ 3'3-diaminobenzine tetrahydrochloride for five minutes. After a light counterstaining with methyl green the preparations were mounted using a solution of veronal buffered glycerol. Control stainings using pre-immune sera were always negative.

Immunohistochemical staining patterns were assessed according to the staining positivity, intensity, and continuity, and were classified as follows: (i) thick and discontinuous, (ii) thin and discontinuous, (iii) fragmentary, and (iv) unrecognisable. Tumour histol- 
ogy was reviewed and the degree of differentiation was classified according to the outline proposed by Oota and Sobin ${ }^{15}$ - that is, well differentiated, moderately differentiated, and poorly differentiated adenocarcinoma. Immunohistochemical and histological correlations were analysed contrasting the serial sections stained with haematoxylin and eosin with those stained for laminin and collagen type IV in each serial triplet.

\section{Results}

Similar patterns were obtained with antisera against laminin and collagen type IV using two contiguous sections. The basement membranes with intense stainings in the normal gastric mucosa showed a thick, continuous line beneath the epithelia. No essential differences were noted between the gastric mucosa and the intestinal metaplastic mucosa in the basement membranes. Vessel walls were also labelled, and prominent staining occurred when the epithelial basement membranes were closely apposed to the blood vessels.

In malignant tissue pericellular stainings of the basement membranes for laminin and collagen type IV showed different patterns in different parts of the adenocarcinoma. The typing of immunohistochemical staining was therefore based on the predominant appearance.

Thick and discontinuous basement membranes featured predominantly in 12 cases; basement membranes were as thick as those of normal epithelia but had occasional disruptions (fig 1); thin and discontinuous basement membranes were detected in 26 cases in which glandular structures of the tumour cells were surrounded by a thinner and occasionally disrupted line of the basement membranes (fig 2); fragmentary basement membranes, characterised by tiny fractions of the stainings adhering to the irregular nests of tumour cells (fig 3 ) or glandular structures were seen in 36 . The remaining 11 showed tumour tissue without recognisable basement membranes.

The relation between the staining patterns of basement membranes and the degree of differentiation of gastric adenocarcinoma is shown in table 1 . In well differentiated adenocarcinomas the thickness of the basement membranes diminished where pleomorphic nuclei, loss of polarity, or papillary structures predominated. Expression of tumoural basement membrane antigens tended to decrease in areas with severe infiltration of inflammatory cells (fig 4) as well as in less well differentiated tumours. In poorly differentiated adenocarcinoma basement membranes stained positive, though this was fragmentary, where the tumour tended to form solid nests or to be composed of gland-like structures. Examples of tumours
Table 1 Relation between staining pattern of basement membranes and degree of differentiation of tumour

\begin{tabular}{lrrrrr}
\hline & \multicolumn{2}{c}{ Degree of differentiation } & \\
\cline { 2 - 4 } & Well & Moderately & Poorly & Total \\
\hline Thick and discontinuous & 12 & 0 & 0 & 12 \\
Thin and discontinuous & 12 & 14 & 0 & 26 \\
Fragmentary & 0 & 15 & 21 & 36 \\
Unrecognisable & 0 & 0 & 11 & 11 \\
\hline
\end{tabular}

without recognisable basement membranes were poorly differentiated adenocarcinomas in which tumour cells grew individually or in small clusters of loosely attached cells. Regardless of histological type, distinctive cells located at the periphery of the tumours generally lacked pericellular staining of basement membranes, although these cells rarely contained diffuse and granular intracellular staining of laminin and collagen type IV.

Lymphatic and blood vessel permeations by tumour cells were often observed in the tumours of this series. Clusters of tumour cells floating in the vessels usually lacked pericellular basement membrane staining. There were, however, six cases with intense, pericellular depositions of laminin in the intravascular clusters (fig 5 ). In these cases the primary tumours were well $(n=4)$ and moderately $(n=2)$ differentiated adenocarcinomas, falling in the thick or thin discontinuous basement membrane categories. Pseudoglandular collections of tumour cells floating in the blood vessels or lymphatics were surrounded by fragmentarily stained laminin. These features were not evident for collagen type IV. Metastasised lymph nodes were studied in 23 cases. The staining features of basement membrane antigens were essentially similar to those in their respective primary tumours.

Eighty three of the 85 patients were followed up for five years or longer (table 2). Of 11 with a tumour belonging to the unrecognisable basement membrane group, only three survived for five years or more, the five year survival being $27 \%$. Conversely, in patients with a tumour in one of the other three categories, each five year survival exceeded $60 \%$.

\section{Discussion}

Malignant tumour cells are essentially characterised by a loss of intact basement membranes. This has been shown by Barsky et al, ${ }^{9}$ who concluded that profound changes of basement membrane expression occur during the transition from a benign lesion to invasive carcinoma in the breast, skin, pancreas and prostate. Recent reviews by Forster et $a^{111^{13}}$ showed that the presence of basement membranes containing laminin in rectal adenocarcinoma correlated with the 


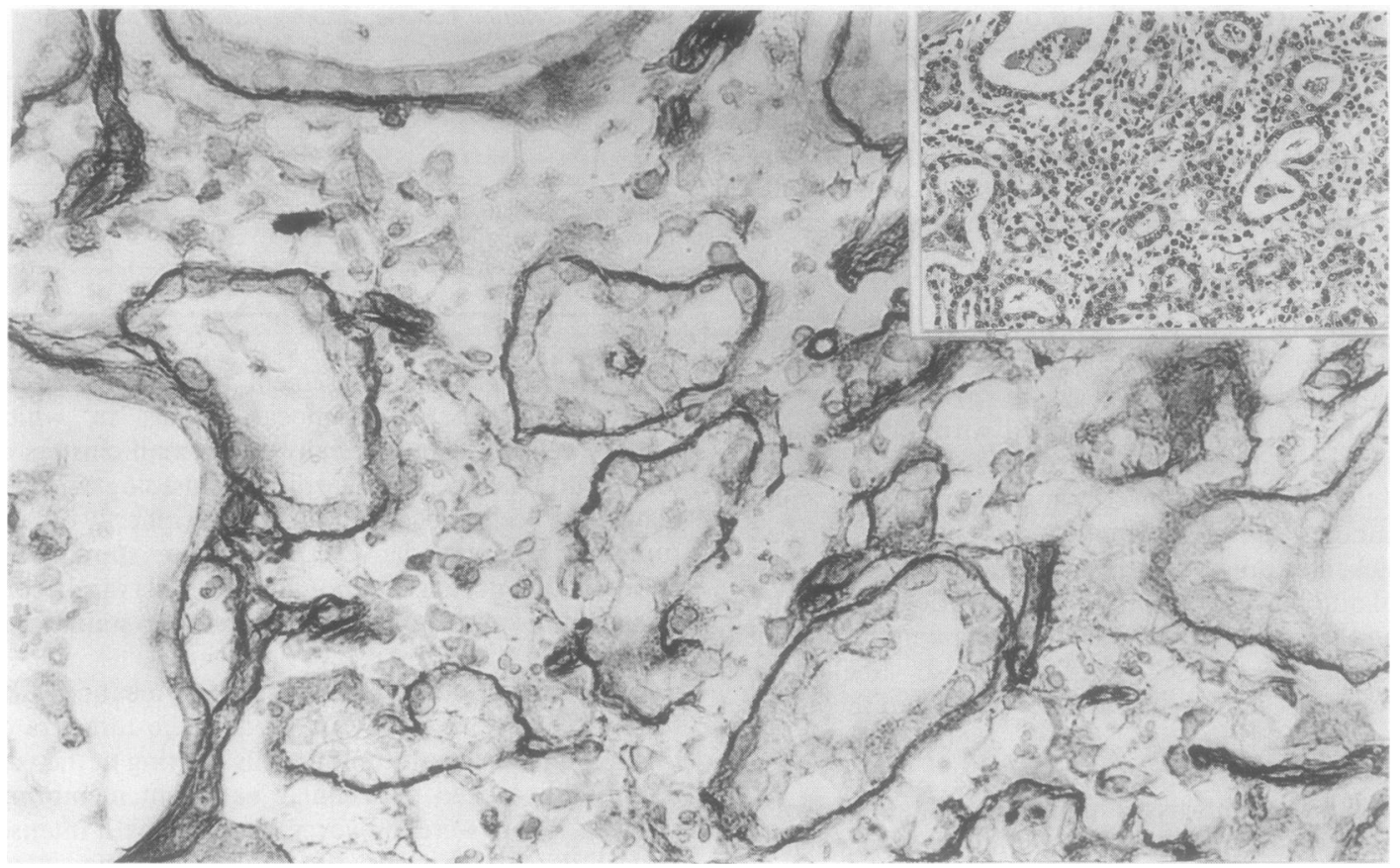

Fig 1 Thick and discontinuous basement membranes around malignant tubules, stained with antiserum for laminin. (Avidin-biotin-peroxidase methyl green.) Inset: low power view of the same area. (Haematoxylin and eosin.)

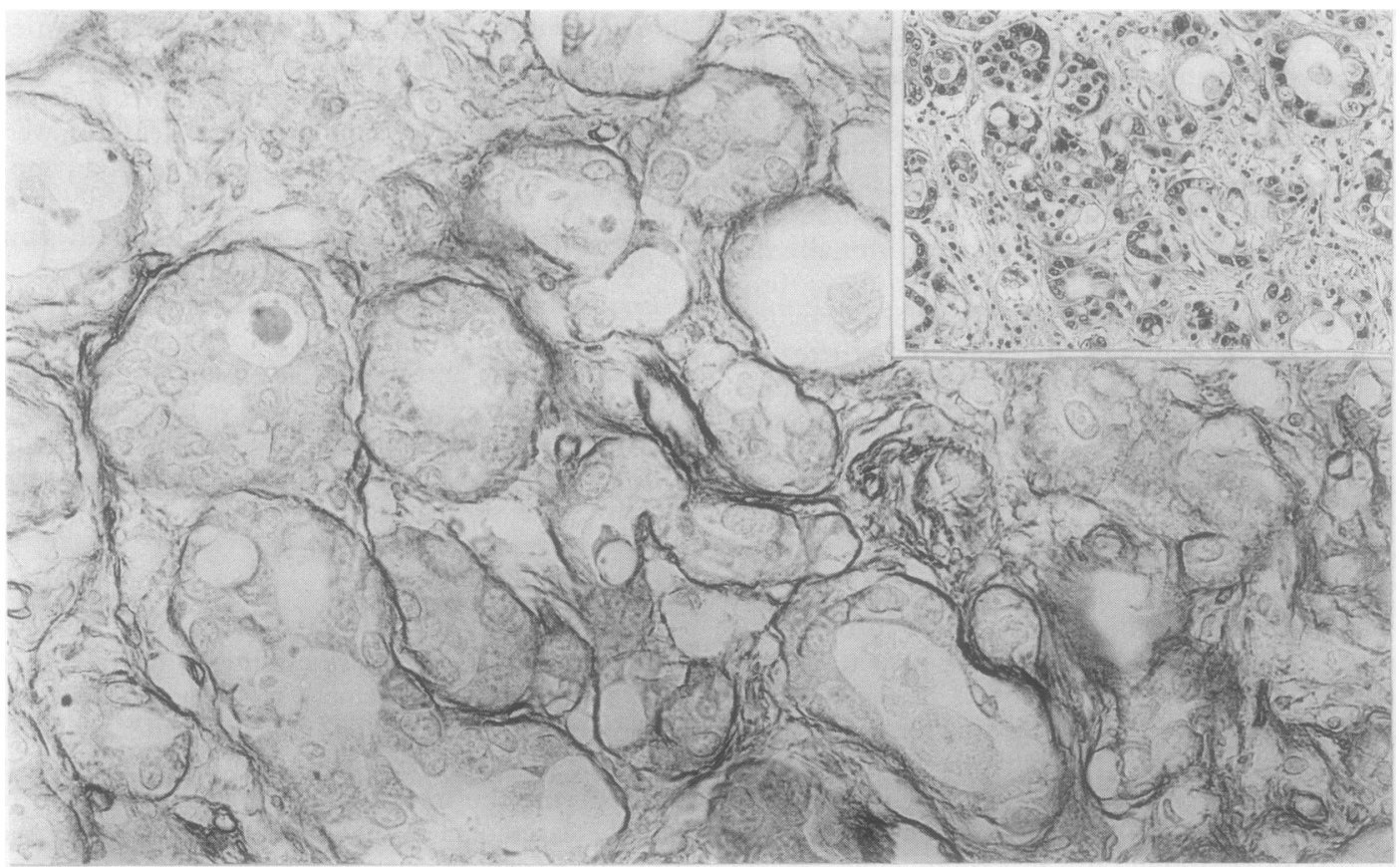

Fig 2 Thin and discontinuous basement membranes attached to malignant tubules of moderately differentiated adenocarcinoma, stained with antiserum for collagen type IV. (Avidin-biotin-peroxidase methyl green.) Inset: low power view of the same area. (Haematoxylin and eosin.) 


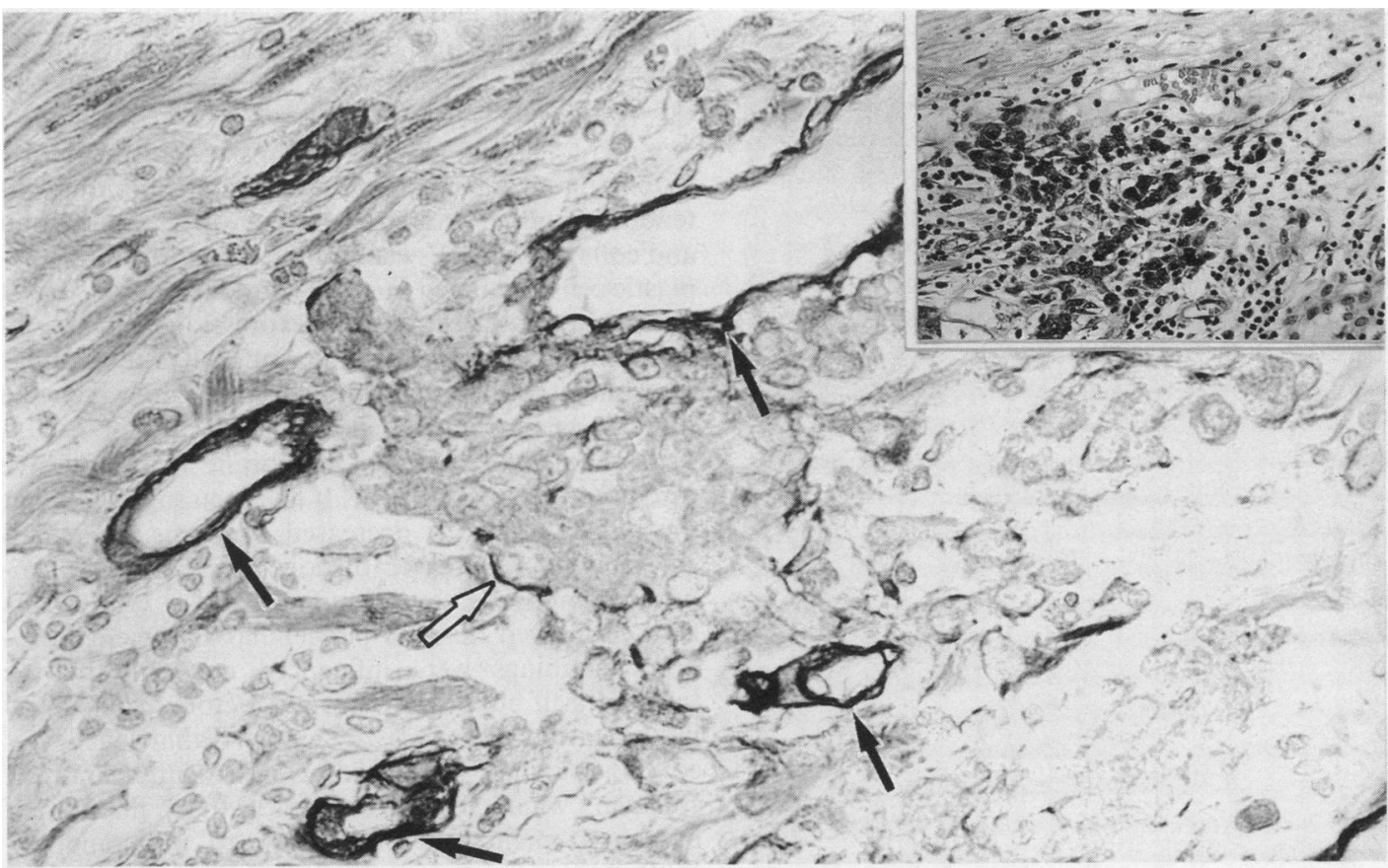

Fig 3 Fragmentary basement membranes as staining fractions (white arrow) adjacent to nest of poorly differentiated adenocarcinoma, stained with antiserum for laminin. Vessel walls were intensely labelled (black arrows).

(Avidin-biotin-peroxidase methyl green.) Inset: low power view of the same area. (Haematoxylin and eosin.)

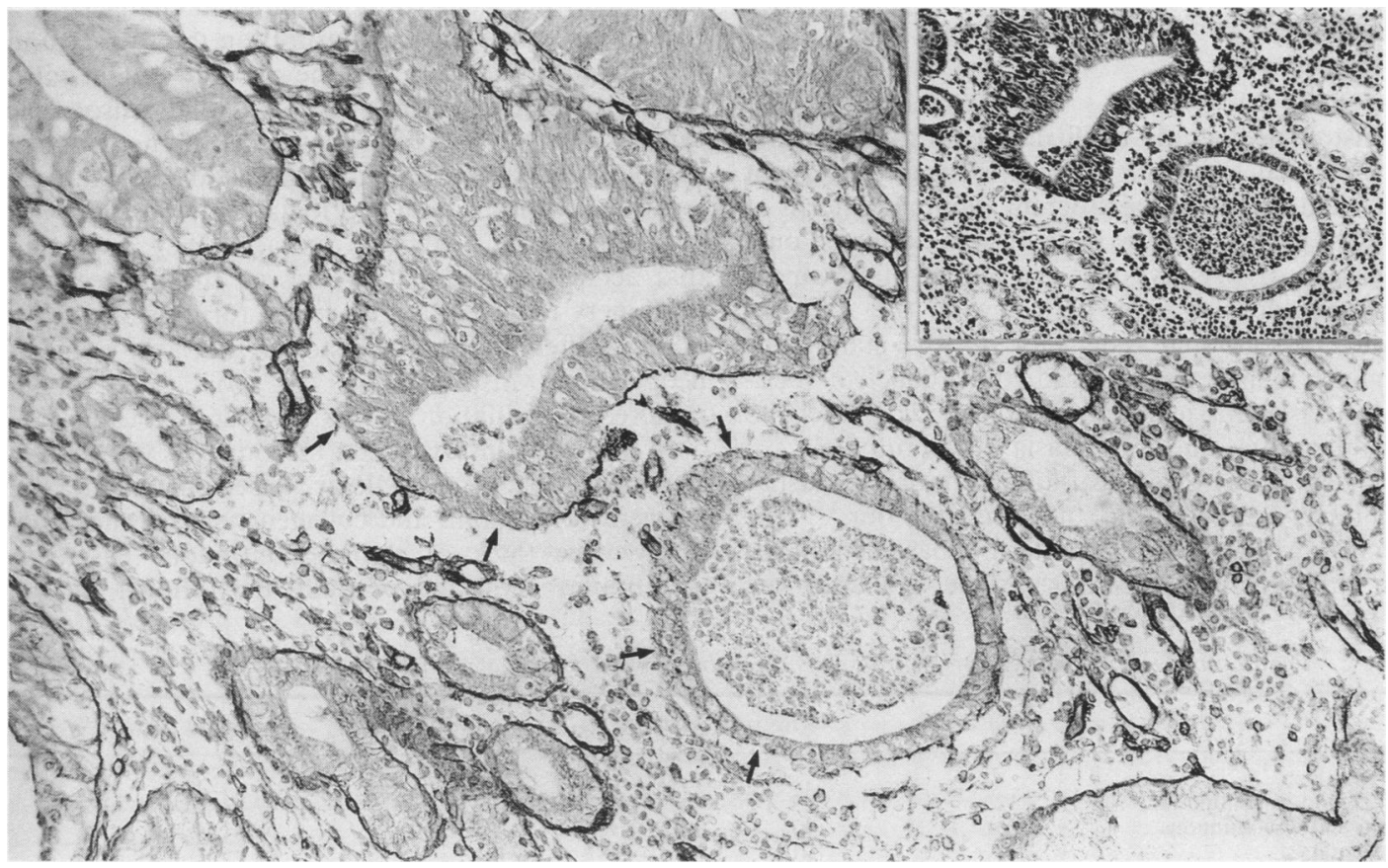

Fig 4 Decreased basement membrane staining (arrows) around inflamed crypt of section of well differentiated adenocarcinoma, stained by antiserum for laminin. (Avidin-biotin-peroxidase methyl green.) Inset: low power view of the same area. (Haematoxylin and eosin.) 


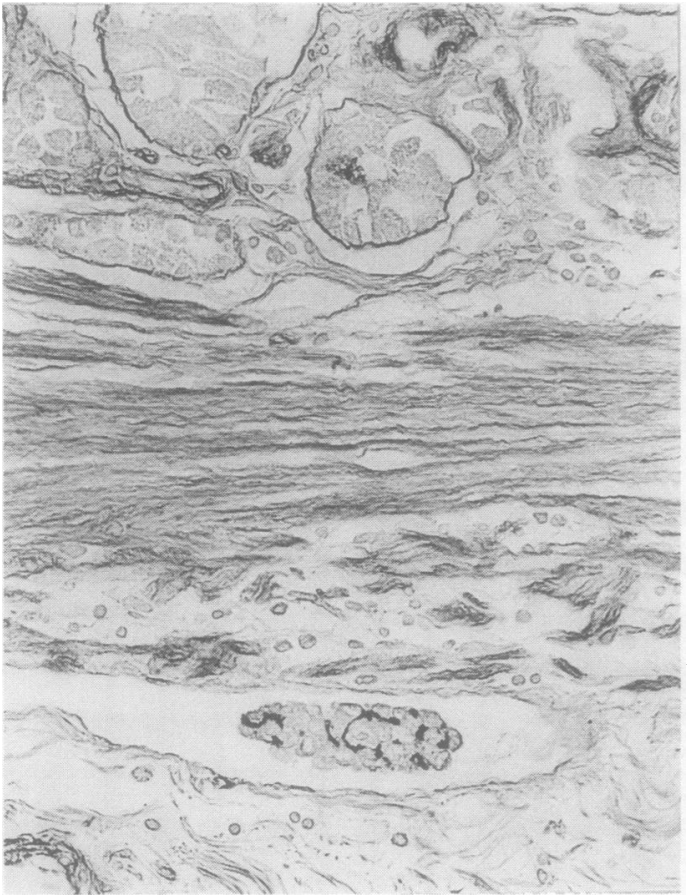

Fig 5 Pericellular deposits of laminin in aggregates of tumour cells floating in submucosal lymphatic vasculature at the bottom. Normal epithelia were surrounded by linear basement membranes at the top. (Avidin-biotin-peroxidase methyl green.)

low histological grade (well differentiated tumours) and increased survival. These investigators did not differentiate between the different staining patterns of basement membrane: the staining was expressed only as positive or negative and the extent of basement membrane development varied with the different tumour. Our grading system for positive staining of basement membrane showed that deficient pericellular basement membranes are related largely to poor differentiation or anaplasia of the tumour.

The degree of histological differentiation in gastric carcinomas varies within the same tumour. ${ }^{15}$ Our observations show that the pattern of basement membrane staining also varied in the same way according to the histological degree of differentiation. Grigioni et $\mathrm{al}^{\mathbf{1 6}}$ reported that the basement membrane staining was related to the local architectural arrangement of the neoplastic cells. In our study the fragmentary basement membranes appeared in areas of the poorly differentiated adenocarcinoma where the tumour cells tended to adhere and the intracytoplasmic laminin and collagen type IV were visible in the invading anaplastic cells, arranged in small strands or cords. These observations suggest that the expression of basement membranes depends on the intensity of cellular attachment rather than on the degree to which the tumour cells have invaded the tissue.

Mechanism of basement membrane loss in invasive carcinoma remains unclear. It has been suggested that the loss is due to a decreased synthesis, decreased assembly, and increased turnover of basement membrane components by the tumour cells. ${ }^{1}$ Our results further showed that the pericellular basement membrane stainings were influenced not only by the tumour itself but also by stromal factors, particularly inflammation. Uitto et $\mathrm{al}^{17}$ reported that leucocytes contain enzymes of degrading basement membrane collagen, which may account for the reduction of basement membrane in relation to inflammation. Infiltration of inflammatory cells into the stroma affects the distribution of basement membranes as well as the structure or shape of tumour cells. Acutee or active inflammation of tissues of the gastro intestinal tract leads to morphological changes (of a regenerative or dysplastic nature) on the surface and pit lining epithelium. ${ }^{1819}$ The in vivo and in vitro studies by Ingber et $a l^{2021}$ showed that anisotropic tumour cells of pancreatic acinar carcinoma, when in contact with basement membranes, had a reorganised location, form, and orientation, intracellular organelles, and a cell-cell contact. They presumed that acinar tumour cells possessed the intracellular machinery required to change the form needed for a normal differentiation of histology but as a result of neoplastic transformation had lost the trigger required for initiation of this structural cascade.

In this study intravascular tumour cells were rarely accompanied by pericellular basement membrane antigens for laminin and collagen type IV and, only rarely were thick or thin discontinuous basement membranes deposited pericellularly, shown by the

Table 2 Relation between staining pattern of basement membranes and five year survival

\begin{tabular}{lllll}
\hline & No & Five year survivals & Unknown & Five year survival \% \\
\hline Recognisable: & & & & \\
$\quad$ Thick and discontinuous & 12 & 8 & 1 & 67 \\
$\quad$ Thin and discontinuous & 26 & 17 & 1 & 68 \\
Fragmentary & 36 & 21 & 64 & 60 \\
Unrecognisable & 11 & 3 & 27 & 6 \\
\hline
\end{tabular}


staining patterns for laminin but not for collagen type IV. These findings suggest that both laminin and collagen type IV would have a role similar to that of basement membranes if the tumour cells were accompanied by anchorage stroma, and that laminin alone would sometimes appear where the epitheliumstroma interface was lost. Nevertheless, the possibility of technical artefact cannot be ruled out because of the relatively small number studied and of the few published.

The prognostic factors of gastric carcinoma were studied by Okada et $a l^{22}$ who found that depth of penetration, invasion of the duodenum, growth pattern of the cancer, lymphatic or vascular invasion, and fibrosis around the cancerous area were clinically important factors. The most pertinent finding in our study was that tumours with unrecognisable basement membranes carried a significantly lower five year survival than tumours with recognisable basement membrane groups for which the five year survival exceeded $60 \%$. Therefore, the tumours can be divided into two groups on this basis.

This study was presented in part at the 75th Annual Meeting of the Japanese Pathological Society on 10 April 1986, Sendai. We thank M Ohara for comments on the manuscript.

\section{References}

1 Liotta LA, Rao CN, Barsky SH. Tumor invasion and the extracellular matrix. Lab Invest 1983;49:636-49.

2 Martinez-Hernandez A, Amenta PS. The basement membrane in pathology. Lab Invest 1983;48:656-77.

3 Birembaut P, Caron Y, Adnet JJ. Usefulness of basement membrane markers in tumoral pathology. $J$ Pathol 1985;145:283-96.

4 Kellokumpu I, Ekblom P, Scheinin TM, et al. Malignant transformation in human colorectal mucosa as monitored by distribution of laminin, a basement membrane glycoprotein. Acta Pathol Microbiol Immunol Scand (Sect A) 1985;93:285-91.

5 Haglund C, Nordling S, Roberts PJ, et al. Expression of laminin in pancreatic neoplasms and in chronic pancreatitis. Am J Surg Pathol 1984;8:669-76.

6 Laurie G, Leblond C, Martin G. Localization of type IV collagen, laminin, heparan sulfate proteoglycan, and fibronectin to the basal lamina of basement membranes. $J$ Cell Biol 1982;95:340-4.

7 Albrechtsen R, Nielsen M, Wewer U, et al. Basement membrane changes in breast cancer detected by immunohistochemical staining for laminin. Cancer Res 1981;41:5076-81.

8 Burtin P, Chavanel G, Foidart JM, et al. Antigens of the basement membrane and the peritumoural stroma in human colonic adenocarcinomas: An immunofluorescence study. Int J Cancer 1982;30:13-20.

9 Barsky SH, Siegal GP, Jannotta F, et al. Loss of basement membrane components of invasive tumours but not by their benign counterparts. Lab Invest 1983;49:140-7.

10 Cam Y, Bellon G, Poulin G, et al. Distribution of type IV collagen in benign and malignant epithelial proliferations. An indirect immunofluorescence study on the breasts, the lungs and the skin. Invas Metast 1984;4:61-72.

11 Forster SJ, Talbot IC, Critchley DR. Laminin and fibronectin in rectal adenocarcinoma: Relationship to tumour grade, stage and metastasis. Br J Cancer 1984;50:51-61.

12 d'Ardenne AJ, Kirkpatrick P, Wells CA, et al. Laminin and fibronectin in adenoid cystic carcinoma. J Clin Pathol 1986;39:138-44.

13 Forster SJ, Talbot IC, Clayton DG, et al. Tumour basement membrane laminin in adenocarcinoma of rectum: an immunohistochemical study of biological and clinical significance. Int J Cancer 1986;37:813-7.

14 Hsu SM, Raine L, Fanger H. Use of avidin-biotin-peroxidase complex (ABC) in immunoperoxidase techniques: a comparison between $\mathrm{ABC}$ and unlabelled antibody (PAP) procedures. J Histochem Cytochem 1981;29:577-80.

15 Oota K, Sobin LH. Histological typing of gastric and oesophageal tumours. International histological classification of tumours.No 18. Geneva: WHO, 1977.

16 Grigioni WF, Biagini G, Errico AD, et al. Behaviour of basement membrane antigens in gastric and colorectal cancer: Immunohistochemical study. Acta Pathol Jpn 1986;36:173-84

17 Uitto V-J, Schwartz D, Veis A. Degradation of basementmembrane collagen by neutral proteases from human leukocytes. Eur J Biochem 1980;105:409-17.

18 Owen DA. Gastritis and duodenitis. In: Appelman HD, ed. Pathology of the esophagus, stomach, and duodenum. New York Churchill Livingstone, 1984:37-77.

19 Riddell RH, Goldman H, Ransohoff DF, et al. Dysplasia in inflammatory bowel disease: Standardized classification with provisional clinical applications. Hum Pathol 1983;14:931-68.

20 Ingber DE, Madri JA, Jamieson JD. Neoplastic disorganization of pancreatic epithelial cell-cell relations: Role of basement membrane. Am J Pathol 1985;121:248-60.

21 Ingber DE, Madri JA, Jamieson JD. Basement membrane as a spatial organizer of polarized epithelia: exogenous basement membrane reorients pancreatic epithelial tumour cells in vitro. Am J Pathol 1986;122:129-39.

22 Okada M, Kojima S, Murakami M, et al. Human gastric carcinoma: Prognosis in relation to macroscopic and microscopic features of the primary tumor. JNCI 1983;71:275-9.

Requests for reprints to: Dr M Enjoji, Second Department of Pathology, Faculty of Medicine, Kyushu University 60, Maidashi 3-1-1, Higashi-ku, Fukuoka 812, Japan. 